Research paper

\title{
Spherical montmorillonite-supported nano-silver as a self-sedimentary catalyst for methylene blue removal
}

\author{
Ning Wang ${ }^{\mathrm{a}, \mathrm{b}, *}$, Fengyan Xiao ${ }^{\mathrm{a}, \mathrm{c}}$, Jinlin Zhang ${ }^{\mathrm{c}}$, Hangsheng Zhou ${ }^{\mathrm{d}}$, Yawei Qin ${ }^{\mathrm{d}}$, Dawei Pan ${ }^{\mathrm{a}, \mathrm{b}, *}$ \\ ${ }^{a}$ CAS Key Laboratory of Coastal Environmental Processes and Ecological Remediation; Research Center for Coastal Environmental Engineering and Technology of \\ Shandong Province; Yantai Institute of Coastal Zone Research, Chinese Academy of Sciences, Yantai, Shandong Province 264003, P.R. China \\ ${ }^{\mathrm{b}}$ Center for Ocean Mega-Science, Chinese Academy of Sciences, 7 Nanhai Road, Qingdao 266071, P.R. China \\ ${ }^{\mathrm{c}}$ Henkel Loctite (China) Co. Ltd, P.R. China \\ ${ }^{\mathrm{d}}$ CAS Key Laboratory of Engineering Plastics, Institute of Chemistry, Chinese Academy of Sciences, Beijing 100190, P.R. China
}

\section{A R T I C L E I N F O}

\section{Keywords:}

Silver nanoparticles

Spherical montmorillonite

Self-sedimentary

Catalyst

Methylene blue

\begin{abstract}
A B S T R A C T
Supported metal nanoparticles using various substrates have been proven as a highly efficient approach for solving the problems of aggregation and recyclability of metal nanoparticles. However, the reusability procedure often involved the abundant devices, which obviously increased the cost and heavily limited the large-scale application of metal nanoparticles. In this work, spherical montmorillonite was used firstly as the substrate for supporting silver nanoparticles on its surface through polydopamine chemistry method. The loading of silver nanoparticles with $40 \mathrm{~nm}$ in diameter was $15.2 \mathrm{wt} \%$ and the specific surface area of this prepared spherical montmorillonite supported silver nanoparticles catalyst was $45.3 \mathrm{~m}^{2} / \mathrm{g}$, giving the catalyst an optimized apparent reduction rate constant $k$ of $1.22 \mathrm{~min}^{-1}$ for the reduction of methylene blue. Furthermore, the prepared catalyst with quickly self-sedimentary property in aqueous solution were conveniently recovered and reused without any devices involves. The spherical morphology and catalytic performance of prepared catalyst were almost unaltered after 5 cycles. Our research aims at opening a new avenue to easily realize the reusability of silver nanoparticles through using the substrate with the self-sedimentary property.
\end{abstract}

\section{Introduction}

Silver nanoparticles (Ag NPs) have been extensively investigated and proven as an efficient catalyst for reduction of organic dyes (Guo et al., 2017; Liu et al., 2017; Shen, Duan, Guo, Zhao, and Xu, 2015). The key issues of recovery and reuse of these $\mathrm{Ag}$ NPs have been significantly considered with respect to the their costs. The substrates including polymer materials (Cao et al., 2017; Xu et al., 2017; Zhu, Wang, Meng, and Diao, 2013), carbon materials (Ji et al., 2016), silica materials (Dong et al., 2014; Khrizanforov et al., 2018), cellulose materials (Torkamani and Azizian, 2016), clay materials (Arora, Mehta, Mishra, and Basu, 2018; Zhang et al., 2018), and metal oxides (Xiao et al., 2018; Sedghi, Asadi, Heidari, and Heravi, 2017) have been fully functionalized through various methods and empolyed to support Ag NPs. This supported approach is now emerging as the most effective strategy to address this issue (Nasrollahzadeh, Atarod, Jaleh, and Gandomirouzbahani, 2016). Among these substrates, recently, magnetic nanoparticles have received greatly attention due to their easyrecovered property from wastewater containing organic dyes through an external magnetic field (Kurtan, Baykal, and Sözeri, 2015; Xie et al., 2014; Yao et al., 2014). Besides, the stable substrates with large surface area for highly loading Ag NPs and strong organic dyes adsorbed capacity were enormously expected due to the increased opportunity for the contacting between Ag NPs and organic dyes to enhance the catalytic performance of the fabricated catalyst (Jeon et al., 2013). These kinds of substrates such as graphene oxide and clay can be recovered through filtration or centrifugation. Until now, despite solving this issue in the laboratory-scale, approaches through the added extra separation and recovery process have yet to be promoted to industrial scale. A major shortcoming of these supported-Ag NPs catalyst is abundant devices involved during the reuse process, which heavily increases the cost of the catalytic procedure and is not well suitable for the industrial applications.

As we know, these substrates with higher density than the wastewater can slowly sink, which can deposit and be recovered from the bottom for some time. Based on the self-sedimentation phenomenon, the substrates with high density and quick sinking ability in wastewater are highly desirable and it is very meaningful to solve an engineering

\footnotetext{
* Corresponding authors.

E-mail addresses: nwang@yic.ac.cn (N. Wang), dwpan@yic.ac.cn (D. Pan).
} 
matter in a physical way. Montmorillonite (Mt) with a mean density of $2-3 \mathrm{~g} / \mathrm{cm}^{3}$ is a natural nanomaterial, can strongly adsorb organic dyes and is considered as a promising candidate as a self-sedimentary substrate (Rafatullah, Sulaiman, Hashim, and Ahmad, 2010). In our previous work, Mt was used as the substrate to in situ supported Ag NPs through polydopamine chemistry method (Wang, Hu, and Zhang, 2017). Although the prepared Mt supported Ag NPs exhibited excellent catalytic performance and good recyclability, their large-scale applications are still restricted by the problem of recyclability during the catalytic procedure since the deposited rate of catalyst is limited by the hydration and swelling of Mt and thus abundant expensive recovered devices are involved. Therefore, the fabricated catalyst with quick sedimentary property is highly desirable.

Spray-drying process has been proposed to realize the consolidating nanoparticles into macroscopic compacts (Stunda-Zujeva, Irbe, and Berzina-Cimdina, 2017; Nandiyanto and Okuyama, 2011). After dissolving the nanoparticles, the solution is passed through atomization, solvent evaporation, drying, and particle collection process to form the final product. Spherical montmorillonite (SMt) (Yawei et al., 2011), silica (Yu et al., 2008), $\mathrm{TiO}_{2}$ (Suh, Jang, Suh, and Suslick, 2010), and $\mathrm{ZrO}_{2}$ (Widiyastuti, Wang, Lenggoro, Iskandar, and Okuyama, 2007) with sub-micrometer size to micrometer size were successfully fabricated through controlling the spray-drying process condition. Here, we fabricated a supported-Ag NPs catalyst using the SMt as the substrate. Polydopamine (PDA) was used here as a coating to modify the surface of SMt and played as a reducing agent role to deposit Ag NPs on the surface of SMt. The model organic dye methylene blue (MB) was selected to estimate the catalytic performance. In addition, the recovery and reusability of the prepared catalyst were evaluated. To the best of our knowledge, this is the first report of fabricated SMt-supported Ag NPs using as a catalyst for reduction of MB, paving a new avenue to greatly economically solve an engineering problem in a physical way and exhibiting the great prospect in practical treatment of wastewater containing organic dyes.

\section{Materials and methods}

\subsection{Instruments and reagents}

Chemicals and reagents were all obtained from Sinopharm Chemical Reagent Co. Ltd., China and directly used as received. The general formula of montmorillonite is (Na, $\mathrm{K}, \mathrm{Ca})_{0.33}\left(\mathrm{Al}_{1.67} \mathrm{Mg}_{0.33}\right)$ $\mathrm{Si}_{4} \mathrm{O}_{10}(\mathrm{OH})_{2} \cdot n \mathrm{H}_{2} \mathrm{O}$. Na-montmorillonite (Mt) used in our research is kindly provided by Zhejiang Fenghong Co. Ltd. and used without further purification. The cation exchange capacity (CEC) of Na-montmorillonite is about $80 \mathrm{cmol}(+) / \mathrm{kg}$. Samples were characterized with scanning electron microscopy (SEM, S-4800, HITACHI, Japan), X-ray diffraction (XRD, Bruker D8-Advantage powder diffractometer, Germany), and $\mathrm{N}_{2}$ adsorption (ASAP2020, US). The Ag content was measured through an inductively coupled plasma mass spectrometry (ICP-MS, ELAN DRC II, Perkin Elmer, Hong Kong). In addition, the catalytic process was recorded through an UV-Vis spectrometer (PERSEE UT 1810, China).

\subsection{Granulation of $M t$}

Spray-drying technique was employed to fabricate spherical Mt. In general, $5.0 \mathrm{~g}$ of Mt was mixed with $98 \mathrm{~mL}$ of ethanol and $2 \mathrm{~mL} \mathrm{H}_{2} \mathrm{SO}_{4}$ (98\%) under $60 \mathrm{~min}$ sonication to obtain the slurry. The addition of acid can greatly clean and disaggregate the Mt and thus increase the number of micropores and the specific surface area (Myriam, 1998). The slurry was fed into a spray-dryer (YC-015, Pilotech Instrument \& Equipment Co. Ltd., China). The condition was $60 \mathrm{~mL} \mathrm{~min}^{-1}$ feed rate, $105^{\circ} \mathrm{C}$ drying temperature, and 0.8 atm compressed air supply pressure. The yield was around $50 \%$.

\subsection{Fabrication of SMt-supported Ag NPs}

Spherical Mt-supported Ag NPs (Ag@SMt) was synthesized through modifying the Mt with polydopamine (PDA), followed by depositing Ag NPs on the PDA modified SMt. Generally, $1.0 \mathrm{~g}$ of dopamine (DA) was added into $200 \mathrm{~mL}$ DI water containing $10.0 \mathrm{~g}$ of SMt and $20 \mathrm{mM}$ Tris$\mathrm{HCl}$ buffer. The $\mathrm{pH}$ was adjusted to 8.5. After shake at room temperature for $6 \mathrm{~h}$, the PDA modified SMt was filtered from the suspension and then washed with alcohol for at least three times. Then PDA modified SMt (PDA@SMt) was further dispersed in $50 \mathrm{~mL} 100 \mathrm{mM} \mathrm{AgNO}_{3}$ aqueous solution under dark with $12 \mathrm{~h}$ rotator (MX-RL-Pro, SCILOGEX, USA). After filtration, the brown microspheres were dried at $70{ }^{\circ} \mathrm{C}$ for at least $12 \mathrm{~h}$ through vacuum oven to obtain the final product Ag@SMt.

\subsection{Adsorption and catalytic experiment}

About $20 \mathrm{mg}$ of sample and $100 \mathrm{~mL}$ of $100 \mathrm{mg} \mathrm{L}^{-1} \mathrm{MB}$ aqueous solution were mixed under constant stirring. The adsorption process of MB was monitor by UV-vis absorption spectra. The removal percentage of MB was calculated following the Eq. (1) (Vimonses, Lei, Jin, Chow, and Saint, 2009):

$\mathrm{R}_{\mathrm{MB}}=\left(\frac{\mathrm{C}_{0}-\mathrm{C}_{\mathrm{t}}}{\mathrm{C}_{0}}\right) \times 100 \%$

where $\mathrm{R}_{\mathrm{MB}}$ is the reduction efficiency of $\mathrm{MB}$, and $C_{\mathrm{t}}$ and $C_{\mathrm{o}}$ are the concentration of the dyes at time $t$ and 0 , respectively.

\subsection{Catalytic reduction of $M B$}

The catalytic performance of the Ag@SMt was evaluated by its catalytic reduction capacity towards MB. Typically, $2 \mathrm{mg}$ catalyst, dye solution ( $1 \mathrm{~mL}$ of $250 \mathrm{mg} \mathrm{L}^{-1}$ ), and $\mathrm{NaBH}_{4}(2 \mathrm{~mL}$ of $0.1 \mathrm{M}$ ) aqueous solutions were mixed with $7 \mathrm{~mL}$ DI water under stirring. The reduced process of MB was monitored at $665 \mathrm{~nm}$ using a UV-vis spectrometer. After the whole reduction process was completed, the self-sedimentary Ag@SMt catalyst was easy recovered from the bottom of the solution and then reused. The key factors such as the concentrations of catalyst, organic dye, and reduce agent were fully investigated.

\section{Results and discussion}

\subsection{Synthesis and characterization of Ag@SMt}

The spray-drying technology is widely used in practical uses, which has been fully proved by its application in the manufacturing of inorganic materials (Park, Kang, Kwon, and Park, 2018). The main principle of this technology is to deliver and rapidly heat the slurry via the direct injection of very small droplets. Spherically shaped particles are typically generated using the spray-drying method because the spherical form is the most stable structural morphology (Vehring, 2008). Therefore, SMt in our experiment was prepared through this technology and used as the substrate for supporting Ag NPs. In addition, the strong adhesive property of polydopamine (PDA) and excellent reduced capacity towards $\mathrm{Ag}$ precursor have extensively investigated (Fu et al., 2015; Liu, Ai, and Lu, 2014). Hence spherical Mt-supported Ag NPs (Ag@SMt) was facile fabricated through PDA chemistry method, as shown in Scheme 1.

To prove the successful preparation of Ag@SMt, SEM was employed to observe the morphologies of spherical Mt (SMt), PDA coated SMt (PDA@SMt), and Ag@SMt, respectively. As shown in Fig. 1a, SMt prepared by the spray-drying technique presents a typical spherical morphology with an average diameter of $25 \mu \mathrm{m}$ and its spherical morphology does not change under the PDA coated process (Fig. 1b). After the Ag-supported process, small bright nanoparticles with a mean diameter of $40 \mathrm{~nm}$ are well observed on the surface of PDA@SMt 


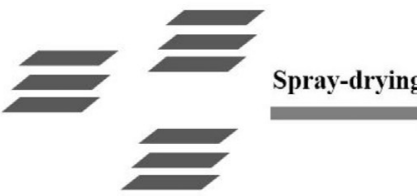

Montmorillonite (Mt)

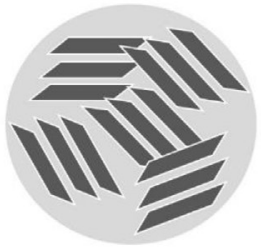

Spherical Mt (SMt)

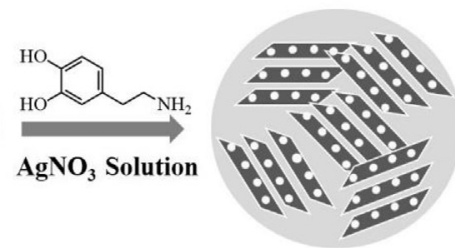

Ag@SMt

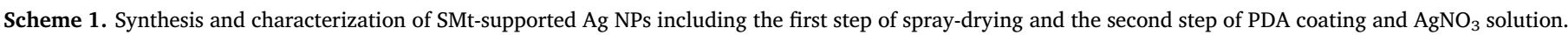

(Fig. 1c) and are further examined as Ag NPs by XPS exhibited in Fig. 1d. These results demonstrate that Ag NPs can be uniformly dispersed on the PDA modified SMt surface and the prepared Ag@SMt can maintain a stable spherical morphology even through long time coating and supported process. The curves of $\mathrm{N}_{2}$ adsorption-desorption isotherm of samples are shown in the inset of Fig. 1. SMt with a typical mesoporous structure is observed. The specific surface area and total pore volume are calculated as $60.2 \mathrm{~m}^{2} / \mathrm{g}$ and $0.37 \mathrm{~cm}^{-3} / \mathrm{g}$, which is much higher than those of pristine Mt $\left(31.8 \mathrm{~m}^{2} / \mathrm{g}\right.$ and $0.26 \mathrm{~cm}^{-3} / \mathrm{g}$, Fig. $\mathrm{S} 1$ and Table 1), indicating the effective spherical-made process. After the coated with PDA, the specific surface area and total pore volume slightly decrease $54.2 \mathrm{~m}^{2} / \mathrm{g}$ and $0.36 \mathrm{~cm}^{-3} / \mathrm{g}$. Compared with the SMt, after the Ag-supported process, the specific surface area of Ag@SMt further decreases to $45.3 \mathrm{~m}^{2} / \mathrm{g}$, while the total pore volume obviously decreased to $0.30 \mathrm{~cm}^{-3} / \mathrm{g}$. Combined with Ag NPs content on Ag@SMt which is calculated to be $15.2 \mathrm{wt} \%$ from the quantified by ICP/MS, the dramatic decreases of specific surface area and the total pore volume are mainly due to the occupation of large amount of Ag NPs on SMt surface (Wang et al., 2017).

To investigate the effect of granulation process, PDA coated process, and Ag-supported process on Mt, XRD was performed to measure the dspacing of all the samples. Fig. 2 exhibits that the d-space of SMt is increased to $1.54 \mathrm{~nm}$ after acid treatment, indicating the acid dissolves the octahedral layer (Myriam, 1998). Upon the coated process, the dspace further increases to $1.95 \mathrm{~nm}$, suggesting the thickness of PDA coated on SMt is about $0.21 \mathrm{~nm}$. However, d-space is almost unaltered while the (001) reflection has low intensity after the Ag-supported
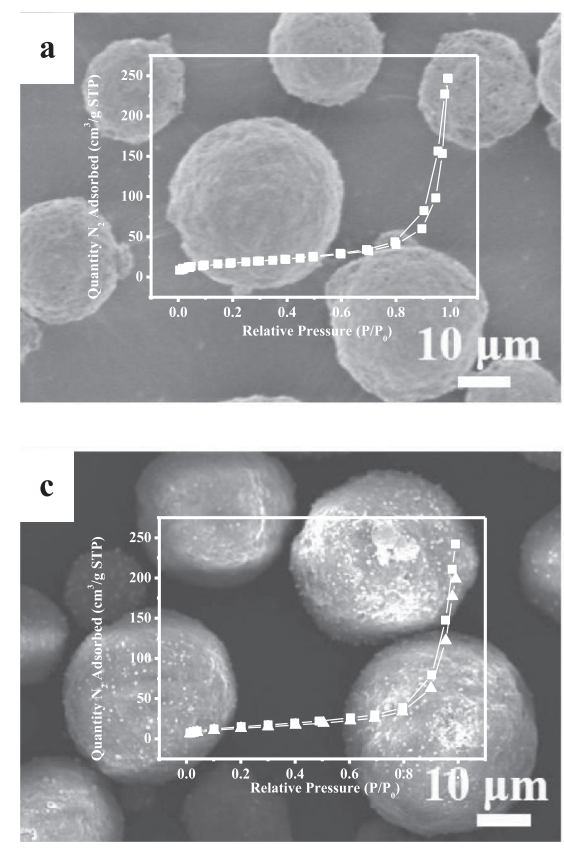

Table 1

Mesoscale properties of Mt, SMt, PDA@SMt and Ag@SMt.

\begin{tabular}{lll}
\hline Sample & BET surface area $\left(\mathrm{m}^{2} / \mathrm{g}\right)$ & Pore volume $\left(\mathrm{cm}^{3} / \mathrm{g}\right)$ \\
\hline Mt & 31.8 & 0.26 \\
SMt & 60.2 & 0.37 \\
PDA@SMt & 54.2 & 0.36 \\
Ag@SMt & 45.3 & 0.30 \\
\hline
\end{tabular}

process, illustrating that delamination happened and most Ag NPs grew on the external surface of SMt, which is well consistent with the reported pristine Mt supported Ag NPs in our previous work (Wang et al., 2017). In addition, four peaks at $2 \theta$ of $38.1^{\circ}, 44.4^{\circ}, 64.4^{\circ}$, and $77.8^{\circ}$ appear, which should be assigned to the (111), (200), (220), and (311) crystal faces of Ag NPs (fcc, JCPDS card no. 04-0783), confirming the successfully supported Ag NPs on the SMt surface (Guo et al., 2017). Furthermore, we used the Scherrer equation $D h k l=k \lambda / \beta \cos \theta$ and calculated the crystallite of the Ag NPs as $0.4 \mathrm{~nm}$. Considering the size of Ag NPs observed from SEM as $40 \mathrm{~nm}$, the Ag NPs are composed by a large amount of crystallite.

\subsection{Adsorption of Ag@SMt}

Recently, most of the researchers have paid attention to organic dyes absorbed capacity of substrates since it is possible to increase the opportunity for the contacting between supported Ag NPs and organic dyes to enhance the catalytic performance of catalysts (Xie et al., 2014).
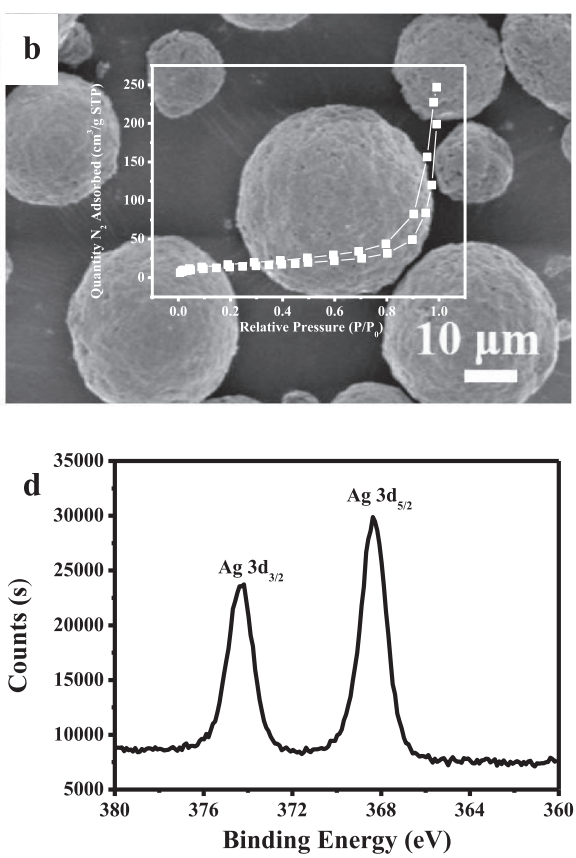

Fig. 1. SEM images of SMt (a), PDA@SMt (b), Ag@SMt microspheres (c) and XPS spectra of Ag@SMt: Ag 3d of Ag@Mt microspheres (d), with inset $N_{2}$ sorption profiles of SMt, PDA@SMt, and Ag@SMt. 


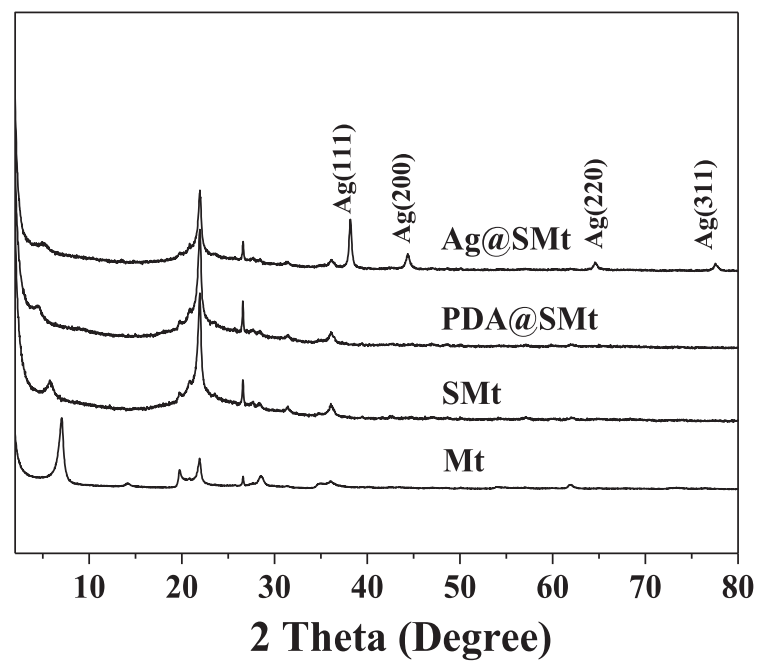

Fig. 2. XRD patterns of Mt, SMt, PDA@SMt, and Ag@SMt.

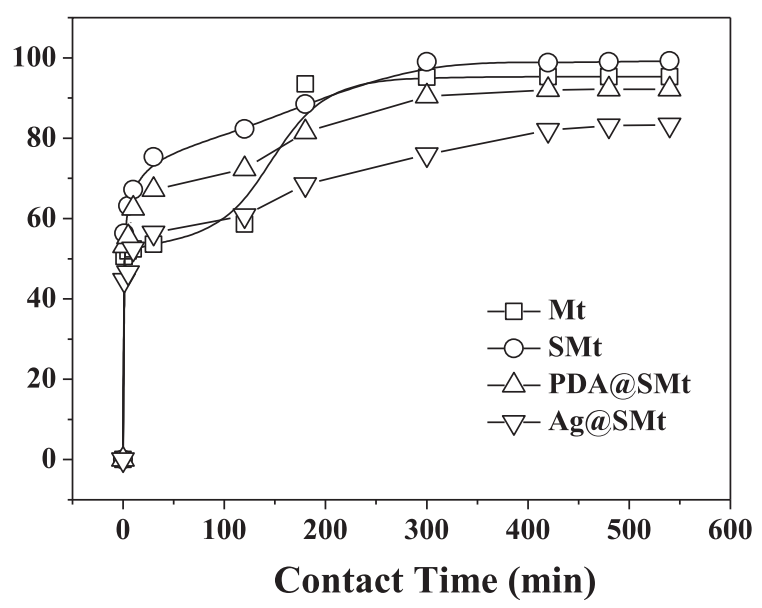

Fig. 3. The adsorption kinetics of MB on Mt, SMt, PDA@SMt, and Ag@SMt.

The zeta potentials of SMt, PDA@SMt, and Ag@SMt at pH6.5 are $-42.5 \mathrm{mV},-38.2 \mathrm{mV}$, and $-33.4 \mathrm{mV}$, respectively, therefore, charge positive dyes methylene blue (MB) was selected as the model. The change of MB during the adsorption process is facile monitored by timedependent UV-Vis absorption spectra due to the band of MB appearing at $665 \mathrm{~nm}$. As shown in Fig. 3, all four samples including Mt, SMt, PDA@SMt, and Ag@SMt present the adsorption behavior with three stages. In the primary stage, the MB quickly adsorbs on the surface of samples, following by the gradual intra-particle diffusion stage and finally reaches the equilibrium stage. The three samples SMt, PDA@SMt, and Ag@SMt exhibit different intra-particle diffusion rate, probably due to the difference of zeta potentials. Among the three samples, SMt and PDA@SMt show the large adsorption of MB with $48.0 \mathrm{mg} \mathrm{g}^{-1}$ and $45.5 \mathrm{mg} \mathrm{g}^{-1}$ at equilibrium stage, respectively, while Ag@SMt decreases to $38.7 \mathrm{mg} \mathrm{g}^{-1}$ because the surface is occupied by great amount of Ag NPs. Furthermore, the MB removal percentage of Ag@SMt decreases to $83.0 \%$ at equilibrium stage, which is $99.2 \%$ and $92.2 \%$ for SMt and PDA@SMt, respectively. In addition, compared with SMt, the gradual intra-particle diffusion rate of pristine Mt is much lower, while the large adsorption of $\mathrm{MB}$ at equilibrium stage is almost the same, reflecting the slower sinking rate of Mt Although the adsorption capacity decreasing upon the Ag-supported process, the value is still much higher than other substrates, such as $\mathrm{Fe}_{3} \mathrm{O}_{4}\left(1.6 \mathrm{mg} \mathrm{g}^{-1}\right.$ ) (Xie et al., 2014) and Zeolite (10.8 $\mathrm{mg} \mathrm{g}^{-1}$ ) (Woolard, Strong, and Erasmus, 2003) reported elsewhere, indicating the efficient adsorption capacity of the prepared catalyst.

\subsection{Catalytic properties of Ag@SMt}

Ag NPs have been proven as an efficient catalyst towards the reduction of organic dyes and the supported Ag NPs exhibited higher catalytic performance than single Ag NPs due to the enhanced adsorption capacity (Guo et al., 2017). Therefore, the catalytic capacity of $\mathrm{Ag} @$ SMt was evaluated through the reduction of MB in the presence of $\mathrm{NaBH}_{4}$. Typically, at the beginning of MB reduction, the peak at $665 \mathrm{~nm}$ decreases its intensity, hence the catalytic reduction process of MB is feasibly monitored by its time-dependent absorbance change using UV-vis spectroscopy. As shown in Fig. 4a, with the prolonged reduction time, the absorbance peak of $\mathrm{MB}$ at $665 \mathrm{~nm}$ rapidly decreases to zero in 15 min with the blue color of MB solution gradually disappears, indicating the degradation of carcinogenic MB to colorless and inactive form leucomethylene blue (Rice, 2001). The catalytic performance of $\mathrm{Ag} @$ SMt catalyst is much related to the valid surface area of Ag NPs for the electron relay from $\mathrm{NaBH}_{4}$ to MB. Therefore, the reaction kinetics of the reduction of the MB under different catalytic conditions are calculated by monitoring their time-dependent UV-Vis absorption spectra. From the linear relation of $\ln \left(\mathrm{C}_{\mathrm{t}} / \mathrm{C}_{0}\right)$ with the prolonged reaction time, the apparent reduction rate constant $(k)$ is received. Fig. $4 \mathrm{~b}$ shows that $k$ increases to $0.98 \mathrm{~min}^{-1}$ with increased $\mathrm{Ag} @ S M t$ concentration when fixing the concentrations of $\mathrm{MB}$ and $\mathrm{NaBH}_{4}$ as $250 \mathrm{mg} \mathrm{L}^{-1}$ and $0.02 \mathrm{M}$, respectively. The increased $k$ is mainly due to the increased valid surface area of Ag NPs (Wunder, Lu, Albrecht, and Ballauff, 2011). Further increasing the concentration of Ag@SMt would not enhance the catalytic performance due to enough surface area providing for electron transfer.

The catalytic mechanism of Ag NPs is based on the electron transfer from the electron donor $\mathrm{NaBH}_{4}$ to electron accepter MB. Therefore, the catalytic reduction process mainly occurs on the Ag NPs surface. Therefore, the competition between the electron donor and the electron acceptor on the available Ag NPs surface would greatly influence the $k$. As shown in Fig. 4c, when fixing the concentrations of $\mathrm{NaBH}_{4}$ and $\mathrm{Ag@}$ SMt as $0.02 \mathrm{M}$ and $0.2 \mathrm{~g} \mathrm{~L}^{-1}$, the $k$ first decreases to $0.20 \mathrm{~min}^{-1}$ as $\mathrm{MB}$ concentration increases to $400 \mathrm{mg} \mathrm{L}^{-1}$, and then unaltered with the further increased $\mathrm{MB}$ concentration. On the contrary, as shown in Fig. 4d, when fixing the concentrations of $\mathrm{MB}$ and Ag@SMt as $250 \mathrm{mg} \mathrm{L}^{-1}$ and $0.2 \mathrm{~g} \mathrm{~L}^{-1}$, the $k$ increases to $0.52 \mathrm{~min}^{-1}$ as the $\mathrm{NaBH}_{4}$ concentration increases to $40 \mathrm{mM}$, and then unchanged with the further increased concentration of $\mathrm{NaBH}_{4}$ to $60 \mathrm{mM}$. It can be understood that $k$ deceases with $\mathrm{MB}$ while increases with $\mathrm{NaBH}_{4}$ concentration under limited Ag NPs surface because the coverage degree of MB and $\mathrm{NaBH}_{4}$ on the Ag NPs surface exist an equilibrium. The increasing of MB results in the increasing of the coverage degree of $\mathrm{MB}$ while decreasing the coverage degree of $\mathrm{NaBH}_{4}$. $k$ keeps a constant at higher concentration of $\mathrm{MB}$ since the coverage degree of $\mathrm{MB}$ and $\mathrm{NaBH}_{4}$ tends to a constant with a large coverage degree of $\mathrm{MB}$ and small coverage degree of $\mathrm{NaBH}_{4}$, vice versa. All these results demonstrate that the $k$ of our synthesized Ag@SMt can be well optimized by tailoring the concentrations of catalyst, dyes, and $\mathrm{NaBH}_{4}$. It is worth to note that the optimized $k$ $\left(1.22 \mathrm{~min}^{-1}\right)$ is much higher than those $\left(0.34 \mathrm{~min}^{-1}\right.$ (Zhu et al., 2013), $0.43 \mathrm{~min}^{-1}$ (Xie et al., 2014), $0.058 \mathrm{~min}^{-1}$ (Tang et al., 2015), and $0.25 \mathrm{~min}^{-1}$ (Sahoo, Kumar, Thiyagarajan, Thakur, and Panda, 2018)) reported in the literature, which can be contributed to the uniform dispersion and high loading of Ag NPs on the SMt with strong adsorption capacity towards organic dyes.

\subsection{Recyclability examination}

Recyclability is the key property of catalyst to maintain its sustainable and efficient performance besides lower its cost for its practical applications. The successive 5 cycles were carried out to determine its recyclability, as shown in Fig. 5a. For one cycle, we first mixed Ag@ 

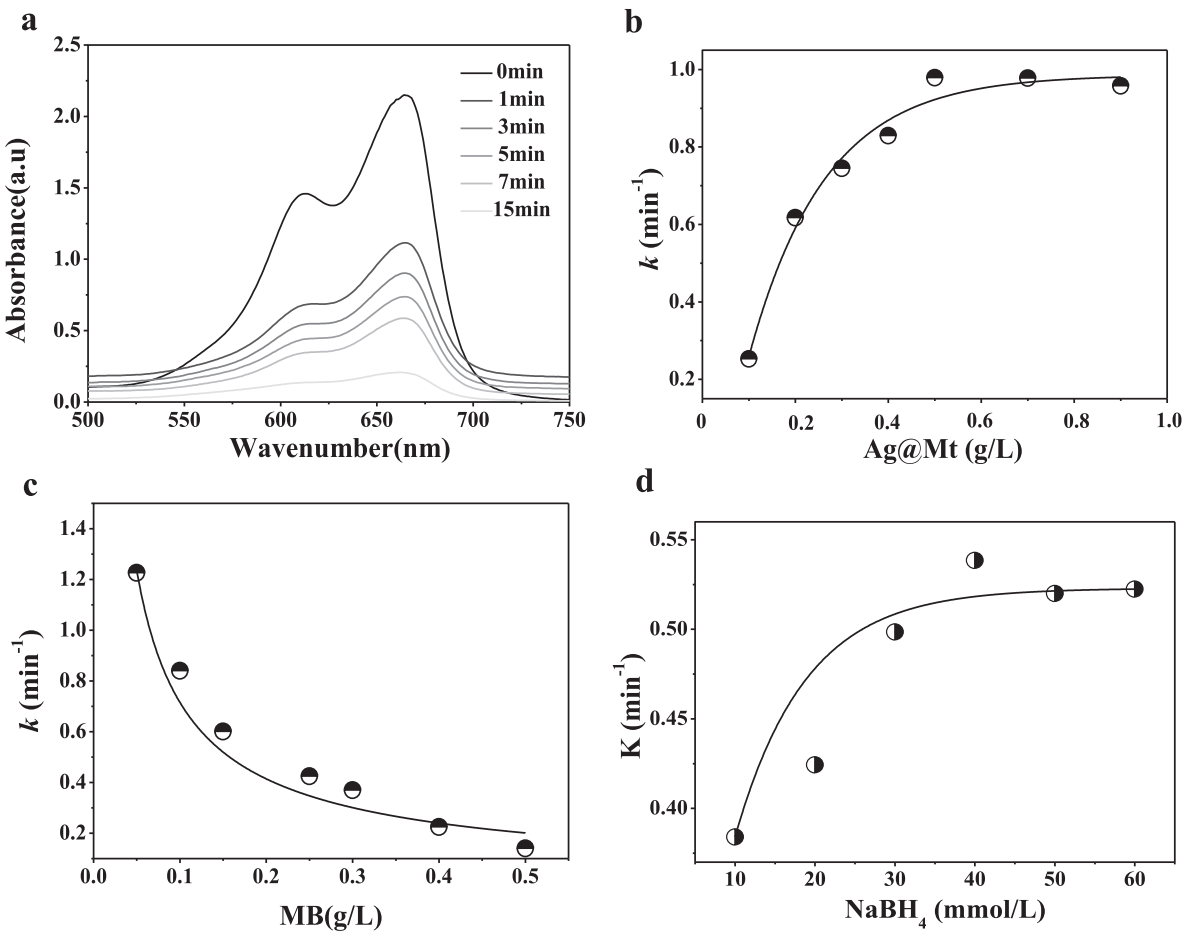

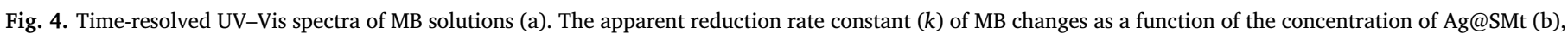
$\mathrm{MB}$ (c), and $\mathrm{NaBH}_{4}$ (d), respectively.

SMt, MB, and $\mathrm{NaBH}_{4}$ together under a shaker, with the prolonged reaction time, the blue color vanishes. Then static settlement for $5 \mathrm{~min}$, followed by pouring the reactive solution. The prepared Ag@SMt exhibits an excellent catalytic performance with unaltered reduction efficiency in the tested five cycles (Fig. 5b). Furthermore, the $k$ slight decreases to $90 \%$ in the 5th cycle (Fig. 5c). Obviously, the self-sedimentary substrate SMt plays an important role in the recovery of $\mathrm{Ag}$
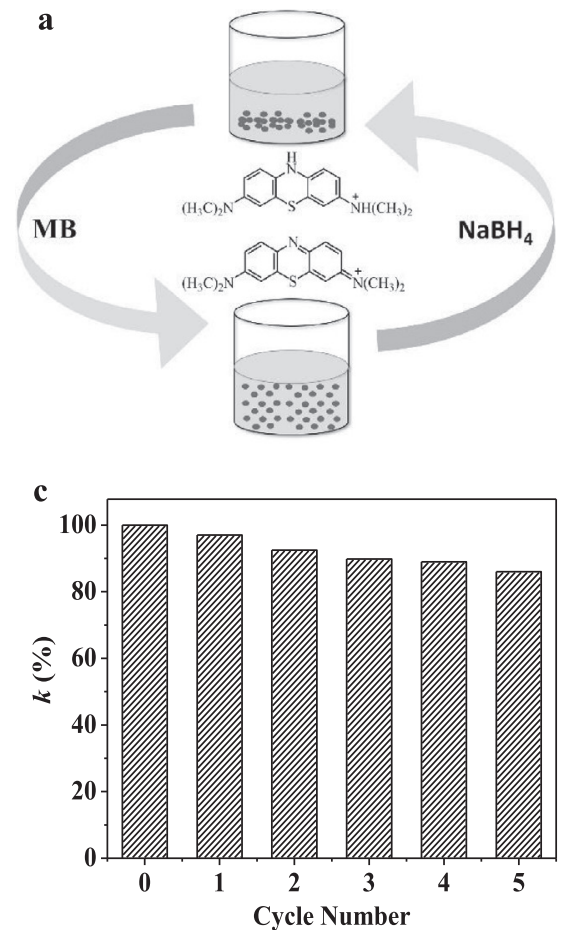

catalyst. The weight loss percentage of Ag@SMt after 1st, 3rd, and 5th cycle is determined to be $3.2 \mathrm{wt} \%, 9.5 \mathrm{wt} \%$, and $12.1 \mathrm{wt} \%$, respectively. In addition, after 5th cycle, as shown in Fig. 5d, the prepared Ag@SMt still maintains spherical morphology with Ag NPs supported on SMt, reflecting the good stability of the prepared catalyst in the aqueous solution.

b

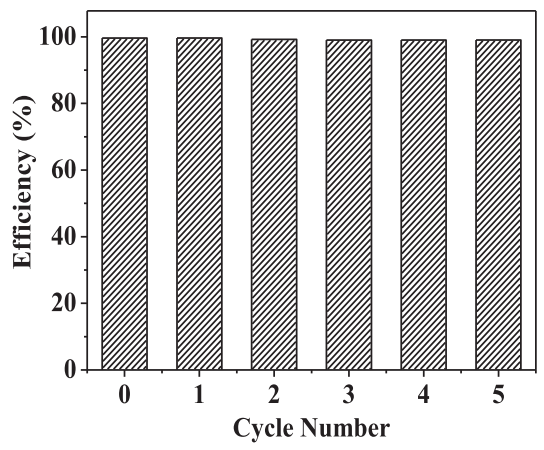

d

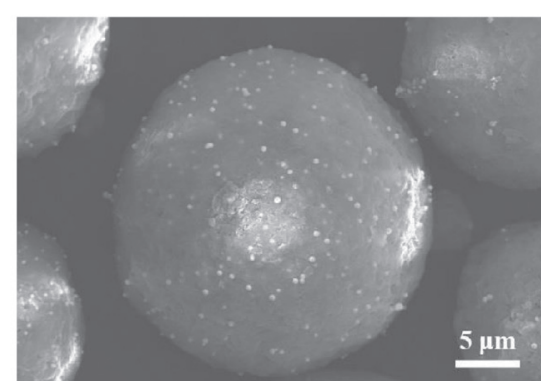

Fig. 5. Scheme of the cycle process of Ag@SMt to catalytic reduction of MB (a), the changes of reduction efficiency (b) and apparent reduction rate constants $k$ of Ag@SMt in the reduction of MB with the prolonged cycle number (c), and SEM images of Ag@SMt microspheres after 5th cycle (d). 


\section{Conclusions}

By fabricating spherical montmorillonite through spray-drying technology, the engineering problem is conveniently solved in a physical way. In this work, we first selected spherical montmorillonite (SMt) as the substrate to in situ support Ag NPs through polydopamine chemistry method. The prepared Ag@SMt with 15.2 wt $\%$ of Ag NPs loading and $45.3 \mathrm{~m}^{2} / \mathrm{g}$ of the specific surface area exhibits an optimized apparent reduction rate constant $k$ of $1.22 \mathrm{~min}^{-1}$ for catalytic reduction of methylene blue. More importantly, the prepared Ag@SMt can be easily recovered and reused without any devices involved due to the self-sedimentary property of SMt. In addition, Ag@SMt shows excellent stability and recyclability for five cycles with unaltered spherical morphology and almost unchanged catalytic performance. Our strategy to solve the engineering issue in a physical way may pave a new avenue to easily realize the application of Ag NPs to the large-scale treatment of wastewater containing organic dyes.

\section{Author contributions}

All authors have given approval to the final version of the manuscript. Ning Wang and Fengyan Xiao contributed equally to this work.

\section{Acknowledgments}

The authors gratefully acknowledge the funding support from National Natural Science Foundation of China (No. 51703110), Key Research and Development Program of Shandong Province (No. 2016ZDJS04B03), Key Science and Technology Program of Yantai City (No. 2018ZHGY075).

\section{Appendix A. Supplementary data}

Supplementary data to this article can be found online at https:// doi.org/10.1016/j.clay.2019.03.021.

\section{References}

Arora, N., Mehta, A., Mishra, A., Basu, S., 2018. 4-Nitrophenol reduction catalysed by AuAg bimetallic nanoparticles supported on LDH: homogeneous vs, heterogeneous catalysis. Appl. Clay Sci. 151, 1-9.

Cao, H.L., Huang, H.B., Chen, Z., Karadeniz, B., Lü, J., Cao, R., 2017. Ultrafine silve nanoparticles supported on a conjugated microporous polymer as high-performance nanocatalysts for nitrophenol reduction. ACS Appl. Mater. Interfaces 9, 5231-5236.

Dong, Z., Le, X., Li, X., Zhang, W., Dong, C., Ma, J., 2014. Silver nanoparticles immobilized on fibrous nano-silica as highly efficient and recyclable heterogeneous catalyst for reduction of 4-nitrophenol and 2-nitroaniline. Appl. Catal. B Environ. 158-159, 129-135.

Fu, J., Chen, Z., Wang, M., Liu, S., Zhang, J., Zhang, J., Han, R., Xu, Q., 2015. Adsorption of methylene blue by a high-efficiency adsorbent (polydopamine microspheres): kinetics, isotherm, thermodynamics and mechanism analysis. Chem. Eng. J. 259, $53-61$.

Guo, Y., Tang, D., Zhang, L., Li, B., Iqbal, A., Liu, W., Qin, W., 2017. Synthesis of ultrathin carbon dots-coated iron oxide nanocubes decorated with silver nanoparticles and their excellent catalytic properties. Ceram. Int. 43, 7311-7320.

Jeon, E.K., Seo, E., Lee, E., Lee, W., Um, M.K., Kim, B.S., 2013. Mussel-inspired green synthesis of silver nanoparticles on graphene oxide nanosheets for enhanced catalytic applications. Chem. Commun. 49, 3392-3394.

Ji, T., Long, C., Mu, L., Yuan, R., Knoblauch, M., Bao, F.S., Zhu, J., 2016. In-situ reduction of Ag nanoparticles on oxygenated mesoporous carbon fabric: exceptional catalyst for nitroaromatics reduction. Appl. Catal. B Environ. 182, 306-315.

Khrizanforov, M., Fedorenko, S.V., Mustafina, A., Kholin, K.V., Nizameev, I.R., Strekalova, S.O., Grinenko, V., Gryaznova, T., Zairov, R., Mazzaro, R., 2018. Silicasupported silver nanoparticles as an efficient catalyst for aromatic C-H alkylation and fluoroalkylation. Dalton Trans. https://doi.org/10.1039/C8DT01090K.

Kurtan, U., Baykal, A., Sözeri, H., 2015. Recyclable $\mathrm{Fe}_{3} \mathrm{O}_{4} @$ Tween20@Ag nanocatalyst for catalytic degradation of azo dyes. J. Inorg. Organomet. Polym. 25, 921-929.

Liu, Y., Ai, K., Lu, L., 2014. Polydopamine and its derivative materials: synthesis and promising applications in energy, environmental, and biomedical fields. Chem. Rev. 114, 5057-5115.

Liu, N., Zhang, W., Li, X., Qu, R., Zhang, Q., Wei, Y., Feng, L., Jiang, L., 2017. Fabrication of robust mesh with anchored $\mathrm{Ag}$ nanoparticles for oil removal and in situ catalytic reduction of aromatic dyes. J. Mater. Chem. A 5, 15822-15827.

Myriam, M., 1998. Structural and textural modifications of palygorskite and sepiolite under acid treatment. Clay Clay Miner. 46, 225-231.

Nandiyanto, A.B.D., Okuyama, K., 2011. Progress in developing spray-drying methods for the production of controlled morphology particles: from the nanometer to submicrometer size ranges. Adv. Powder Technol. 22, 1-19.

Nasrollahzadeh, M., Atarod, M., Jaleh, B., Gandomirouzbahani, M., 2016. In situ green synthesis of $\mathrm{Ag}$ nanoparticles on graphene oxide/ $\mathrm{TiO}_{2}$ nanocomposite and their catalytic activity for the reduction of 4-nitrophenol, Congo red and methylene blue. Ceram. Int. 42, 8587-8596.

Park, J.H., Kang, S.W., Kwon, T.S., Park, H.S., 2018. Spray-drying assisted synthesis of a $\mathrm{Li}_{4} \mathrm{Ti}_{5} \mathrm{O}_{12} / \mathrm{C}$ composite for high rate performance lithium ion batteries. Ceram. Int. $44,2683-2690$.

Rafatullah, M., Sulaiman, O., Hashim, R., Ahmad, A., 2010. Adsorption of methylene blue on low-cost adsorbents: a review. J. Hazard. Mater. 177, 70-80.

Rice, L., 2001. Investigation into the potential of methylene blue and its derivatives to be used in the photodynamic therapy of non-pigmented and pigmented cells. University of Central Lancashire, pp. i (Abstract).

Sahoo, P.K., Kumar, N., Thiyagarajan, S., Thakur, D., Panda, H.S., 2018. Freeze-Casting of Multifunctional Cellular 3D-graphene/Ag Nanocomposites: synergistically Affect Supercapacitor, Catalytic, and Antibacterial Properties. ACS Sustain. Chem. Eng. 6, 7475-7487.

Sedghi, R., Asadi, S., Heidari, B., Heravi, M.M., 2017. TiO 2 /polymeric supported silver nanoparticles applied as superior nanocatalyst in reduction reactions. Mater. Res. Bull. 92, 65-73.

Shen, H., Duan, C., Guo, J., Zhao, N., Xu, J., 2015. Facile in situ synthesis of silver nanoparticles on boron nitride nanosheets with enhanced catalytic performance. J. Mater. Chem. A 3, 16663-16669.

Stunda-Zujeva, A., Irbe, Z., Berzina-Cimdina, L., 2017. Controlling the morphology of ceramic and composite powders obtained via spray drying-a review. Ceram. Int. 43, 11543-11551.

Suh, W.H., Jang, A.R., Suh, Y.H., Suslick, K.S., 2010. Porous, hollow, and ball-in-ball metal oxide microspheres: preparation, endocytosis, and cytotoxicity. Adv. Mater. 18, 1832-1837.

Tang, Y., Wu, T., Hu, B., Yang, Q., Liu, L., Yu, B., Ding, Y., Ye, S., 2015. Synthesis of thermo- and $\mathrm{pH}$-responsive $\mathrm{Ag}$ nanoparticle-embedded hybrid microgels and their catalytic activity in methylene blue reduction. Mater. Chem. Phys. 149-150, 460-466.

Torkamani, F., Azizian, S., 2016. Green and simple synthesis of Ag nanoparticles loaded onto cellulosic fiber as efficient and low-cost catalyst for reduction of 4-nitrophenol. J. Mol. Liq. 214, 270-275.

Vehring, R., 2008. Pharmaceutical particle engineering via spray drying. Pharm. Res. 25, 999-1022.

Vimonses, V., Lei, S., Jin, B., Chow, C.W.K., Saint, C., 2009. Kinetic study and equilibrium isotherm analysis of Congo Red adsorption by clay materials. Chem. Eng. J. 148, 354-364.

Wang, N., Hu, Y., Zhang, Z., 2017. Sustainable catalytic properties of silver nanoparticles supported montmorillonite for highly efficient recyclable reduction of methylene blue. Appl. Clay Sci. 150, 47-55.

Widiyastuti, W., Wang, W.N., Lenggoro, I.W., Iskandar, F., Okuyama, K., 2007. Simulation and experimental study of spray pyrolysis of polydispersed droplets. J. Mater. Res. 22, 1888-1898.

Woolard, C.D., Strong, J., Erasmus, C.R., 2003. Evaluation of the use of modified coal ash as a potential sorbent for organic waste streams. Appl. Geochem. 18, 1279.

Wunder, S., Lu, Y., Albrecht, M., Ballauff, M., 2011. Catalytic activity of faceted gold nanoparticles studied by a model reaction: evidence for substrate-induced surface restructuring. ACS Catal. 1, 908-916.

Xiao, G., Zhao, Y., Li, L., Pratt, J., Su, H., Tan, T., 2018. Facile synthesis of dispersed Ag nanoparticles on chitosan- $\mathrm{TiO}_{2}$ composites as recyclable nanocatalysts for 4-nitrophenol reduction. Nanotechnology 29, 155601.

Xie, Y., Yan, B., Xu, H., Chen, J., Liu, Q., Deng, Y., Zeng, H., 2014. Highly regenerable mussel-inspired $\mathrm{Fe}_{3} \mathrm{O}_{4} @$ polydopamine-Ag core-shell microspheres as catalyst and adsorbent for methylene blue removal. ACS Appl. Mater. Interfaces 6, 8845-8852.

Xu, P., Liang, X., Chen, N., Tang, J., Shao, W., Gao, Q., Teng, Z., 2017. Magnetic separable chitosan microcapsules decorated with silver nanoparticles for catalytic reduction of 4-nitrophenol. J. Colloid Interface Sci. 507, 353-359.

Yao, T., Cui, T., Wang, H., Xu, L., Cui, F., Wu, J., 2014. A simple way to prepare Au@ polypyrrole $/ \mathrm{Fe}_{3} \mathrm{O}_{4}$ hollow capsules with high stability and their application in cat alytic reduction of methylene blue dye. Nanoscale 6, 7666-7674.

Yawei, Q., Ning, W., Yong, Z., Yingjuan, H., Hui, N., Jin-Yong, D., 2011. Fabrication of nanofillers into a granular "Nanosupport" for Ziegler-Natta catalysts: towards scalable in situ preparation of polyolefin nanocomposites. Macromol. Rapid Commun. 32, 1052-1059.

Yu, X., Ding, S., Meng, Z., Liu, J., Qu, X., Lu, Y., Yang, Z., 2008. Aerosol assisted synthesis of silica/phenolic resin composite mesoporous hollow spheres. Colloid Polym. Sci. 286, 1361-1368.

Zhang, J., Yan, Z., Fu, L., Zhang, Y., Yang, H., Ouyang, J., Chen, D., 2018. Silver nanoparticles assembled on modified sepiolite nanofibers for enhanced catalytic reduction of 4-nitrophenol. Appl. Clay Sci. 166, 166-173.

Zhu, M., Wang, C., Meng, D., Diao, G., 2013. In situ synthesis of silver nanostructures on magnetic $\mathrm{Fe}_{3} \mathrm{O}_{4} @ \mathrm{C}$ core-shell nanocomposites and their application in catalytic reduction reactions. J. Mater. Chem. A 1, 2118-2125. 